\title{
A demonstration of particle duality of light
}

Haili Jiang, Zhihai Liu, Qiuhua Sun, Yancheng Zhao

Haili Jiang, Zhihai Liu, Qiuhua Sun, Yancheng Zhao, "A demonstration of particle duality of light," Proc. SPIE 10452, 14th Conference on Education and Training in Optics and Photonics: ETOP 2017, 104523C (16 August 2017); doi: $10.1117 / 12.2270720$

Event: 14th Conference on Education and Training in Optics and Photonics, ETOP 2017, 2017, Hangzhou, China 


\title{
A demonstration of particle duality of light
}

\author{
JIANG Haili, LIU Zhihai, SUN Qiuhua, ZHAO Yancheng \\ (Harbin Engineering University, College of science, Heilongjiang, Harbin, 150001)
}

\begin{abstract}
The need of understanding and teaching about wave-particle duality if light with gets more and more apparent in the background of the attention of modern physics. As early as the beginning of twentieth Century, Einstein dared to "deny" the development of a very perfect light electromagnetic theory, so that the quantum of light can be developed. In 1924, De Broglie put forward wave-particle duality if light to other micro particles and the concept of matter wave, pointed out that all micro particle has wave-particle duality. This is a very abstract concept for students, most college physics teaching all lack of demonstration about particle duality of light. The present article aims to contribute to demonstrate the wave-particle duality of light at the same time using a simple way based on fiber optical tweezers. It is hoped that useful lesson can be absorbed so that students can deepen the understanding of the particle and wave properties of light. To complement the demonstration experiment for this attribute light has momentum.
\end{abstract}

Keywords: Optical radiation pressure; Optical suspension; College physics; Particle duality of light

\section{Introduction}

The need of understanding and teaching about wave-particle duality if light with gets more and more apparent in the background of the attention of modern physics. As early as the beginning of twentieth Century, Einstein dared to "deny" the development of a very perfect light electromagnetic theory, so that the quantum of light can be developed. It was realized that micro particles also have wave-particle duality. This was first conjectured by De Broglie in $1924^{[1-4]}$. De Broglie put forward the concept of matter wave, pointed out that all micro particle has wave-particle duality. This is a very abstract concept for students, most college physics teaching all lack of demonstration about particle duality of light.

We judge new ideas in terms of our research experience about how to demonstrate this odd particle property of the light. The goal of this article is to present a new practical exercise to explain this concept which is not easy to understand. It contributed to demonstrate the wave-particle duality of light at the same time using a simple way based on fiber optical tweezers. It is hoped that useful lesson can be absorbed so that students can deepen the understanding of the particle and wave properties of light. To complement the demonstration experiment for this attribute light has momentum.

2. Demonstration of the particle property of light

\subsection{Basic principle}

The light radiation pressure, also known as the light pressure is a force generated while light irradiate on the object. The generation of light radiation pressure can be explained from two perspectives. From the point of view of electromagnetic wave, the momentum of the charge under the action of the electromagnetic force will change. By momentum conservation law, the momentum of electromagnetic field will change accordingly ${ }^{[5-7]}$. The change of the momentum caused by the interaction between electromagnetic wave and objects are represented by the Poynting vector.

14th Conference on Education and Training in Optics and Photonics: ETOP 2017, edited by Xu Liu,

Xi-Cheng Zhang, Proc. of SPIE Vol. 10452, 104523C · C 2017 ICO, IEEE, OSA, SPIE

CCC code: $0277-786 \mathrm{X} / 17 / \$ 18 \cdot$ doi: $10.1117 / 12.2270720$

Proc. of SPIE Vol. 10452 104523C-1 
On the other hand, from the point of view of photon theory, the radiation pressure of light is the result of photons passing their momentum to the object. The irradiation of a group of continuous photon with momentum onto the object would generate stable pressure named as light pressure. By the theory of photon, photon energy $\mathrm{E}$ and momentum $\mathrm{P}$ should meet following rules:

$$
\begin{gathered}
E=h v=\hbar \omega=h_{\bar{\lambda}}^{c} \\
p=\frac{h v}{c}=\frac{h}{\lambda}=\frac{h k}{2 \pi}=\hbar k
\end{gathered}
$$

Where, $\mathrm{h}$ is Planck constant and $\mathrm{h}=6.626 \times 10-34 \mathrm{~J} \bullet \mathrm{s}, \mathrm{v}$ is frequency and $k$ is wave vector, $c$ is light speed.

Maxwell pointed out that light is an electromagnetic wave, light radiation pressure equal to electromagnetic wave energy flow density which could be described in terms of Poynting vector S:

$$
P=\frac{S}{c}
$$

Make the time averaged for Poynting vector $\boldsymbol{S}$ then we can get the optical radiation pressure

$$
\langle P\rangle=\frac{\langle S\rangle}{c}=\frac{I}{c}
$$

Where $I$ is light intensity.

The actual pressure force $F$ of this beam acting on an object can be expressed as the power of the incident light $P$ and the efficiency of the force $Q$ :

$$
F=Q \frac{n_{1} P}{c}
$$

Where $n_{1}$ is the refractive index of the surrounding medium, $c$ is the speed of light, $P$ is the optical power. The $Q$ value is a dimensionless constant between $0 \sim 2$. The change of momentum per unit time is $n_{1} P / c$ when the light irradiated on a medium with a refractive index of $n_{1}$. In a certain extent the $Q$ value depends on the optical conditions (such as laser focusing angle, wavelength, beam size, polarization state), micro object conditions (such as size, change of shape and refractive index) and environmental conditions (water, air, etc. $)^{[8-9]}$.

Through the above statement, we can estimate the optical pressure of earth's surface is about when sun light is incident on surface of the earth vertically. Compared with the force of ordinary life it is so small that we can't feel it at all. Until 1960s the invention of laser, laser light source has the advantages of high strength, good collimation which let the mechanical effect of light is fully displayed, and so that we can understand the vital light has the basic properties of momentum.

\subsection{System principle}

In this experiment, the laser beam is projected onto the polystyrene spheres in water. The force of the polystyrene sphere is shown in Figure 1.The optical power of the laser diode is used to balance the radiation pressure and gravity, and to realize the suspension of particles. The relationship between the radiation pressure and the optical power could be measured. 


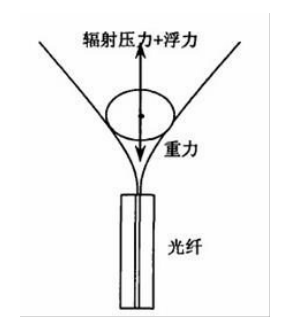

Fig1. Experimental schematic

The fundamental mode Gauss beam propagating along the $z$ axis can be expressed as

$$
\psi_{00}(x, y, z)=\frac{C}{\omega(z)} \exp \left(-\frac{r^{2}}{\omega^{2}(z)}\right) \exp \left\{-t\left[k\left(z+\frac{r^{2}}{2 R}\right)-\arctan \left(\frac{z}{f}\right)\right]\right\}
$$

Where $\omega_{0}$ is the waist radius of the Gauss beam which can be considered as the fiber core with the radius of $9 \mu \mathrm{m}$. The position of the optical fiber is $z_{0}$, the diameter of the polystyrene sphere is 10

$\mu \mathrm{m}$. When the source power is less than a certain limit, the particles will gradually drop. When the light power is $P_{1}$, the particle will suspend at the location of $z_{1}$. The light power increased to $P_{2}$, the propagation direction of movement along the action of light particles in the optical radiation pressure, gradually away from the light source, the light irradiation in the particle decreases again until equilibrium is reached, the particle's position is denoted as $z_{2}$, so we can get

$$
P_{1} \int_{-x-y}^{x} \int_{-y}^{y} \psi\left(x, y, z_{1}\right) d x d y P_{2} \int_{-x-y}^{x} \int_{y}^{y} \psi\left(x, y, z_{2}\right) d x d
$$

The variation law of the light source power and the balance position $z$ can be obtained.

Therefore, in the case of known power source and power distribution, the equilibrium position of the particles, the radiation pressure of the particles in the current physical environment can be calculated directly. 3 Experiment and results

The experimental apparatus is shown in Figure 2.

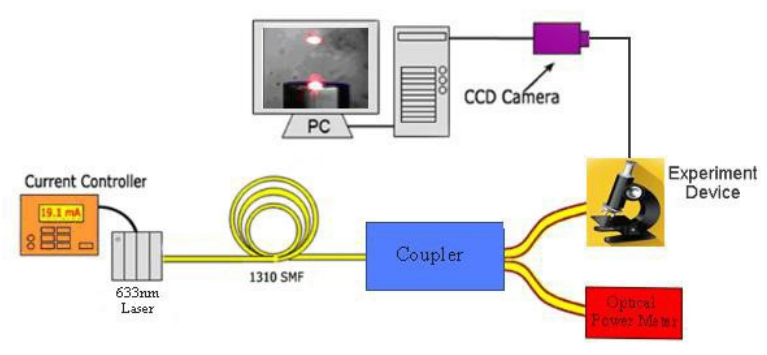

Fig.2 The experimental apparatus 


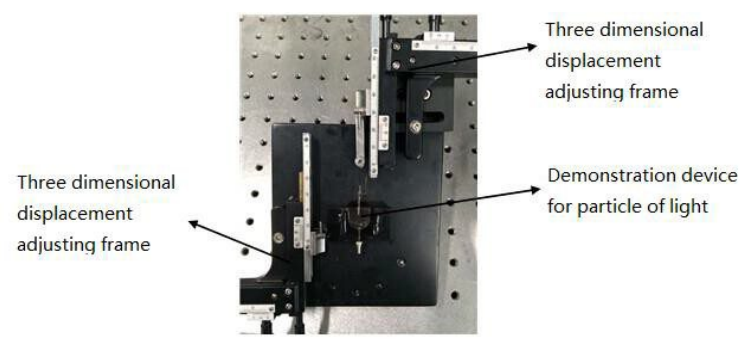

Fig.3 The sample table and capillary effect diagram

The process is as follows: 1) turn on the power of the laser source, adjusting the laser power is $10 \mathrm{~mW}$; 2) injection of purified water to the sample pool with dropper; 3) adjust the sample pool position and the focus of the microscope to make the optical imaging in the sight field; 4) adjust the displacement position, so that it will just set into the optical fiber; 5) the polystyrene spheres suspended droplets on the displacement of tube; 6) when polystyrene appeared in the field of vision, adjust the displacement position of the tube, so that one microspheres just in the fiber output beam; 7) due to the light radiation pressure in the light in the spheres will be suspended in water; 8) to change the optical power so that polystyrene spheres suspended in a different location, record the light power value and the corresponding position; 9) depicts the relation curve of the incident light power and balance position, calculate the radiation pressure of light.

In the experiment, the power of the incident light is recorded and the equilibrium position of the polystyrene spheres is obtained. The data are shown in table 1.

Table 1 The sheet of optical power and equilibrium position

\begin{tabular}{c|c|c|c|c|c|c|c|c|c|c}
\hline $\begin{array}{c}\text { optical } \\
\text { power } / \mathrm{mW}\end{array}$ & 4 & 8 & 12 & 16 & 20 & 24 & 28 & 32 & 36 & 40 \\
\hline $\begin{array}{c}\text { equilibrium } \\
\text { position } / \mu \mathrm{m}\end{array}$ & 29 & 62 & 91 & 123 & 162 & 193 & 233 & 259 & 297 & 327 \\
\hline
\end{tabular}

According to the data in table 1, Figure 4 shows the relationship between the incident light power and the equilibrium position of the particles. 


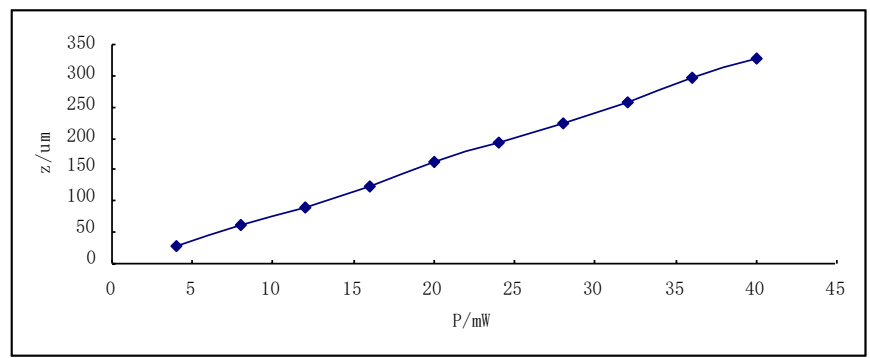

Fig.4 The relationship between the incident light power and the equilibrium position of the

particle

\section{Conclusion}

This paper introduced the particle duality of light in the college physics teaching from the development content of college physics experiment angle. Based on the updating and development of college physics, the principle of Physics - particle duality of light in the application of new technology was highlighted especially the application of the micro field. This new demonstration reflected the new technology the characteristics of teaching of college physics. The students theoretical knowledge of physics to get deeper understanding of the theoretical knowledge and experimental skills of students in the supplement and support each other, to achieve the interaction of perceptual knowledge and rational knowledge of two in the process of cognition, cultivate students' comprehensive ability and creativity.

\section{Reference}

[1]Leung A, Shankar P M, Mutharasan R. A review of fiber-optic biosensors[J]. Sensors and Actuators B: Chemical, 2007,125(2):688-703.

[2]Liu Zhihai, Guo Chengkai, Yang Jun, et al. Single tapered fiber optical tweezers[A]. Proceedings of SPIE[C]. 2007,6534:161-166.

[3]Zhang Yu, Wu Zhongfu, Liu Zhihai, et al. Manip-ulating multi-particles simultaneously with a taper-ed-tip single fiber optical tweezers[A]. Proceed-ings of SPIE[C]. 2009,7280:2L1-2L8. [4]Ashkin A. Acceleration and trapping of particles by radiation pressure[ J]. Physical Review Letters,1970,24(4):156-159.

[5]Ashkin A, Dziedzic J M, Bjorkholm J E, et al. Observation of a single-beam gradient force optical trap for dielectric particles[J]. Optics Letters, 1986,11(5):288-290.

[6] LI Chuang, LIU Zhaoji, WU Bing, et al. High-precision optical fiber end microfabrication system and the experimental research[J].Applied Science and Technology, 2013, 40(2):31-34.

[7] MA Kun, WU Haijun, YANG Jiyong, et al. A fiber seismograph demodulation method based on COSTAS loop[J]. Applied Science and Technology, 2016,43(6):67-71.

[8]Haili Jiang, Yonggui Yuan, Jun Yang, Libo Yuan . A Novel Multi-path Combination Matching Michelson Interferometer for Strain \deformation Sensing, Advanced Sensor Systems and Applications IV,2010.10.18.

[9]Haili Jiang, Yonggui Yuan, Jun Yang, Libo Yuan . The theoretical and experimental study on white light interferometric fiber optic sensors network based on Michelson and Mach-Zehnder interferometers, 2011 Academic International Symposium on Optoelectronics and Microelectronics Technology, AISOMT 2011 2011.10. 